\title{
LOCAL BINARY GRAPH FEATURE REDUCTION FOR THREE-DIMENSIONAL GABOR FILTER BASED HYPERSPECTRAL IMAGE CLASSIFICATION
}

\author{
Mohsen Darvishnezhad ${ }^{1}$, Hassan Ghassemian ${ }^{1, *}$, Maryam Imani ${ }^{1}$ \\ ${ }^{1}$ Image Processing and Information Analysis Laboratory, Faculty of Electrical and Computer Engineering, \\ Tarbiat Modares University, Tehran, Iran \\ Mohsen_darvishnezhad@modares.ac.ir, ghassemi@modares.ac.ir,maryam.imani@modares.ac.ir
}

Commission VI, WG VI/4

KEY WORDS: Hyperspectral, Spectral Features, Spatial Features, Feature Fusion, Three-Dimensional Gabor Filters.

\begin{abstract}
:
One of the challenges of the hyperspectral image classification is the fusing spectral and spatial features. There are several methods for fusing features in hyperspectral image classification. Three-Dimensional Gabor Filters are the best method to extract spectral and spatial features simultaneously. However, one of the problems with using the 3D Gabor filter is the high number of extracted features. In this paper, to reducing extracted features from 3D-Gabor filters and increasing the classification accuracy in hyperspectral images, a novel method named Local Binary Graph (LBG) is used. The LBG method uses a local graph to solve the optimization problem, which maps each pixel to the reduced dimension image and improves the McNemar test result in comparison with the existing methods. Finally, the result of the proposed method achieved $96.2 \%$ and $92.6 \%$ overall accuracy for Pavia University and Indian Pines data set, respectively.
\end{abstract}

\section{INTRODUCTION}

Hyperspectral image classification has been a vibrant area of research in recent years. Given a set of observations, i.e., pixel vectors in a hyperspectral image, classification approaches try to allocate a unique label to each vector of pixels. However, the classification of hyperspectral images has some challenges, such as the presence of redundant features, the limited number of available training samples, and high dimensionality of the data (Kianisarkaleh and Ghassemian, 2016). Imaging spectroscopy (also known as hyperspectral imaging) is an important technique in remote sensing (RS). Hyperspectral (HS) imaging sensors often capture data from the visible through the near-infrared wavelength ranges, thus providing hundreds of narrow spectral channels from the same area on the surface of the earth. These instruments collect data consisting of a set of pixels represented as vectors, which each elements is a measurement corresponding to a specific wavelength. The size of each vector is equal to the number of spectral channels or bands. Hyperspectral images usually consist of several hundred spectral data channels for the same area on the earth's surface; while, in multispectral data, the number of spectral channels is usually up to tens of bands (Ghassemian H., Landgrebe, D., 1988 a and b).

Due to the detailed spectral information available from the hundreds of narrow bands collected by hyperspectral sensors, the accurate discrimination of different materials is possible. This fact makes hyperspectral data a valuable source of information to be fed to advanced classifiers. The output of the classification step is known as the classification map (Zhu, 2017a). Therefore, one of the application from hyperspectral images is the hyperspectral images classification. The common theme in all of these applications is the requirement for classification of each pixel in the scene and reduction of data volume to tractable levels. Classification of a hyperspectral image used to specify the nature of the spectrum of each pixel in the images (Liao et al, 2016a).
There are several methods for HS classification and feature reduction in (Imani and Ghassemian, 2015a). By feature selection or feature extraction, the dimension of features will be reduced. Utilizing an appropriate subset of the original criterion and a search algorithm is needed to feature selection approaches (Zhang et al, 2015a, Li, 2014a). Principle component analysis (PCA) is an unsupervised linear feature extraction method and linear discriminant analysis (LDA) is a supervised linear feature extraction method that both methods will be used widely (Fukunaga, 1990). A linear combination of the original features results in the generated principal uncorrelated components. PCA searches for data with greater variance and preserves them as principle data components. To obtain a good classification, the position of reduced feature space data may be unsuitable to separate properly between classes (Imani and Ghassemian, 2015a). To solve this problem, LDA will be utilized. In this paper, we compare the different methods with proposed method for HS image classification. One of the methods for simultaneously using spectral information along with the spatial information is 3D-Gabor filters but, the problem of using this method is the high number of extracted features. The proposed method in this paper uses Local Binary Graph (LBG) to reducing extracted features from 3D-Gabor filters. Therefore, simultaneously, the dimension reduced and the spatial- spectral features are fused. Experiments on the HS data show the superiority of the proposed method in this paper over other available methods. Finally, the proposed method will be compared with five other methods: feature fusion from LBG, 3DGabor filters, spatial features, spectral features and stacking of the spectral-spatial features.

Section II presents a short analysis of spatial feature extraction. In Section III, we present the local binary graph (LBG), 3DGabor filters and the proposed method. The experimental results on well-known hyperspectral images are presented and discussed in Section IV. Finally, the conclusion is brought in Section V. 

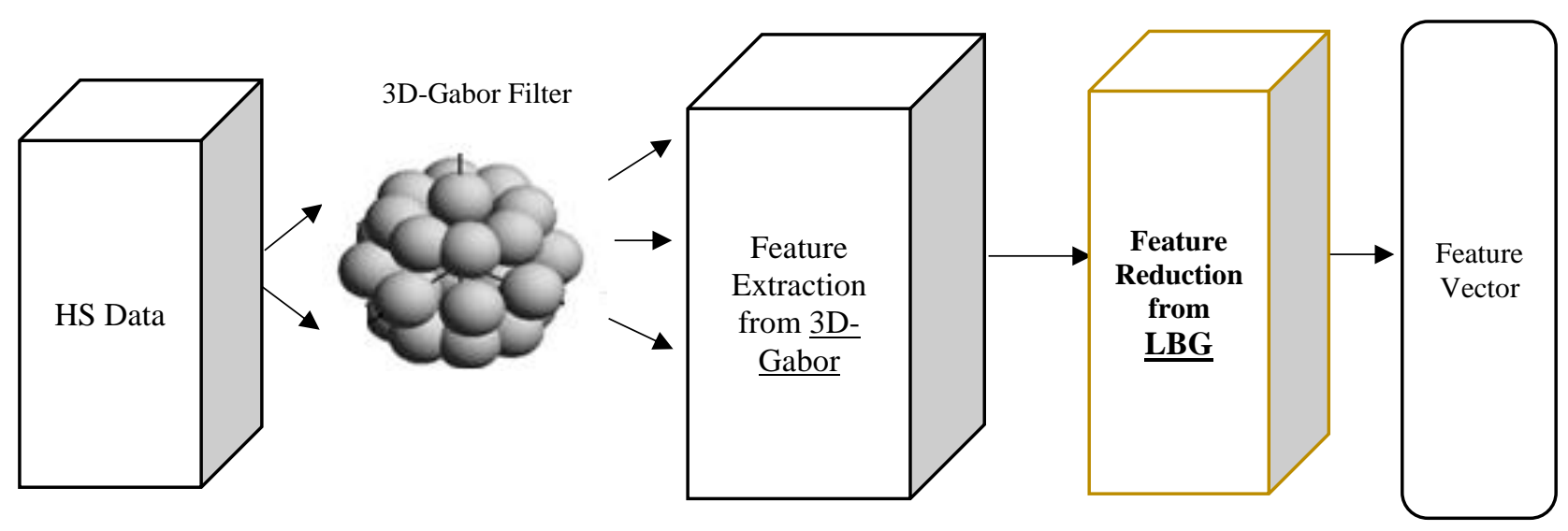

Figure 1. Flowchart of Proposed Method

\section{SPATIAL FEATURE EXTRACTION}

There are many techniques to obtain spatial features of hyperspectral imagery which in this paper, the Morphological Profile feature extraction technique will be used. Typical morphological features used to describe spatial information of very high-resolution remote sensing images, using morphological openings and closings to reconstruction on a graylevel picture and utilizing a structural element of predefined size and shape, will be produced. In the continuation, Morphological image processing will be described totally (Imani and Ghassemian, 2015a, Imani and Ghassemian, 2016).

\section{- Morphological profile (MP)}

Morphological image processing is a set of non-linear elements which is related to the shape or morphology of features in an image, such as edges, frames, etc. After applying a structural element based on opening and closing to a single-band image, the spatial feature of the image is extracted. So, for each pixel $2 \mathrm{~N}+$ 1 spatial feature will be extracted by:

$$
M P_{n}(I)=\left\{\emptyset_{1}(I), \ldots, \emptyset_{n}(I), I, \gamma_{1}(I), \ldots, \gamma_{n}(I)\right\}
$$

where $\emptyset_{i}(I), \gamma_{i}(I)$ with $\mathrm{i}=1,2, \ldots, \mathrm{n}$ are closing and opening by reconstruction operators respectively (Pal, 2010a). The two fundamental operators in Morphological Profiles are erosion and dilation. These operators are applied to an image with a set of known shape, called a structuring element (SE) which results in finding the desired objects in the image. Opening and closing are combinations of erosion and dilation, which opening is to dilate an eroded image to recover as much as possible of the eroded image although closing is to erode a dilated to recover the initial shape of image structures that have been dilated (Chang, 2011a). After the opening/closing affected to the image, image structures with size less than the SE will be removed but the other structures which still present in the image will be modified. Opening and closing by reconstructions are connected operators to avoid detecting fake objects in the image that satisfy the following assertion: If the structure of the image cannot contain the SE, it will be removed, else it will be preserved. For a given SE, geodesic opening or geodesic closing allows knowing the size or shape of some objects present in the image, i.e., the objects that are smaller than the SE will be deleted while the other (that are bigger than the SE) will be preserved (Imani and Ghassemian, 2016, Mirzapour and Ghassemian, 2017).

\section{Proposed Method}

In this section, we proposed a method for reducing spectral and spatial features extraction from 3D-Gabor filters (Fig1). At first, the spatial and spectral features extracted from 3D-Gabor filters (Imani and Ghassemian, 2016). In this step, it was made the 3DGabor filters on the original hyperspectral data sets in order spectral and spatial features extraction together. However, one of the problems with the use of the 3D Gabor filter is the high number of extracted features. To solve this problem, the features can be reduced by local binary graph method analyses (LBG). In the end, the dimensions of the spatial-spectral features will be reduced by LBG. Therefore, the features fusion in lowerdimensional feature space can be obtained (Fig 1).

In the following, the 3D-Gabor filters and Local Binary Graph method will be described exactly.

\subsection{Three-Dimensional Gabor Filters}

A Gabor filter is a sinusoidal function modulated by a Gaussian envelope. A 3-D spectral-spatial Gabor filter is defined in the radiance domain by (2) for a three-dimensional state in the domain of space. Therefore it can be said that a 3D Gabor (Imani and Ghassemian, 2016), is the product of a 3D Gaussian and a $3 \mathrm{D}$ harmonic function. The length of the axes is controlled by the Gaussian and the frequency is controlled by the harmonic function. 3D Gabor wavelets are used for spatiotemporal analysis of a three-dimensional signal, like a video sequence, to extract motion energy features.

$$
\begin{array}{r}
f(x, y, z)=\frac{1}{\sqrt[1.5]{2 \pi} \cdot \sigma^{3}} \cdot e^{\frac{-1}{2 \cdot \sigma^{2}}\left(x^{2}+y^{2}+z^{2}\right)} \\
\times \cos \left(2 \pi\left(f_{x} \cdot f_{y} \cdot f_{z}\right)\right)
\end{array}
$$

Where the variables $x$ and $y$ are the spatial variables, and $z$ is the wavelength variable. Also, $f_{x}, f_{y}, f_{z}$ the following relationships are obtained.

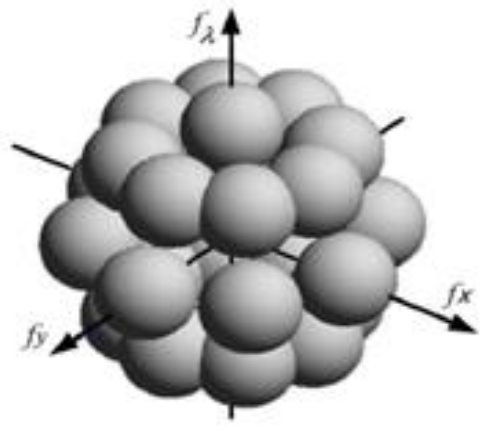

Figure 2. 3D Gabor filters 


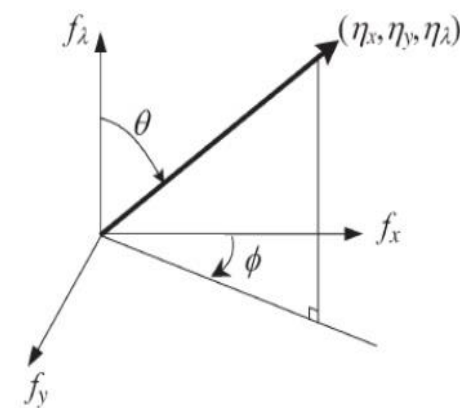

Figure 3. 3D Gabor filters orientation by $\theta, \varphi$

where

$$
\begin{aligned}
& f_{x}=F \sin \theta \cos \varphi \\
& f_{y}=F \sin \theta \sin \varphi \\
& f_{z}=F \cos \theta
\end{aligned}
$$

$\left(f_{x}, f_{y}, f_{z}\right)$ represents the frequency of the sinusoidal component and also the radial center frequency of the filter in the 3-D frequency domain and $\mathrm{F}$ is the central frequency that can be obtained from 6.

$$
F^{2}=f_{x}^{2}+f_{y}^{2}+f_{z}^{2}
$$

By using this filter on the hyperspectral image new features in the new area from (2) will be obtained ( Ghamisi, 2014a).

\subsection{Local Binary Graph}

Applying a simplified stacking spatial-spectral features cannot make much difference between feature sources. A local binary graph (LBG) method (Liao et al, 2016a, Huang, 2013a) is proposed to reduce dimension and fuse features of the spectral information and the spatial information extracted by morphological profiles, simultaneously. In Local Binary Graph, unlike Global Binary Graph, the fusion graph is built on the full data by moving a sliding window from the first pixel to the last one. Because in this method, data fusion is built on randomly selected samples, a clear improvement over a previous approach could be observed. In LBG, at first, Morphological Profile (MP) will be built on the first Principle Components Analysis (which used $99 \%$ of data energy) of the original hyperspectral data sets. Symmetric padding is used to avoid the margin effect when the sliding window is close to the margin of image same way as (Imani and Ghassemian, 2015a, Hussain, 2018a). An MP includes of the original image (one of the PC features) and $\mathrm{M}$ features of the opening operator with SEs that increase in size (all applied on the first PC ) and $\mathrm{M}$ features of the closing operator with the same SE. Every MP extracts $d$ features of spatial-spectral which $d=(2 M$ +1 ) for each pixel. At first, the spatial-spectral features will be normalized between $[0,1]$ and then, these features can be stacked from:

$$
X^{\text {stack }}=\left[X^{\text {spatial }} ; X^{\text {specectral }}\right]
$$

In LBG, the final goal is achieving a transformation matrix "W" to calculate feature fusion in the new space by (7)

$$
z_{i}=W^{T} \cdot x_{i}
$$

Where $x_{i}$ is a multivariate parameter that can be set to $\mathrm{x}_{i}^{\text {stack }}$ and $z_{i}$ is the feature fusion in new space The $\mathrm{W}$ matrix not only fused the different features into the less dimensioned space but also utilizes the local information properties of each pixel's neighbors, hence the LBG method will be reduced the features of the highdimensional features. The transformation matrix $W$ can be defined by $(8)$

$\arg \min \left(\sum_{i, j=1}^{N}\left\|W^{T} \cdot x_{i}^{\text {stack }}-W^{T} \cdot x_{j}^{\text {stack }}\right\|^{2} \cdot A_{i, j}\right) \mid W \in$ $R^{(D+B) \times d}$

where "W" is the transformation matrix and the "A" matrix represents the edges of an undirected graph $G=(X, A)$. This matrix (A) represents a relation between the graph nodes $x_{i}$ and $x_{j}$. In this method, two data points $x i$ and $x j$ result in adjacent (connected) graph nodes if they had "close" distance to each other. Thus, we have $A_{i, j}=1$ if $x_{i}$ and $x_{j}$ are "close" and $A_{i, j}=0$ if $x_{i}$ and $x_{j}$ are "far apart". In the other words, $x_{j}$ will be "close" to $x_{i}$ if it belongs to its k nearest neighbors $(\mathrm{kNN})$. At first, KNN will be determined by calculating the distance between each data point $x i$ and the other data points $x j(\mathrm{j}=1 \ldots \mathrm{N}$, and $\mathrm{i}=\mathrm{j})$, then $\mathrm{kNN}$ sorted the distances ascending and selected the first $\mathrm{K}$ distances. Finally, a graph will be produced between each data point $x i$ with the other data points $x j(\mathrm{j}=1 \ldots \mathrm{N}$, and $\mathrm{i}=\mathrm{j})$, obtained through $\mathrm{kNN}$. The effectiveness of using such graph to fuse multiple feature sources for classification has been discussed in the very recent studies [4]. "A" matrix can be defined by (9):

$A_{i, j}=\left[\begin{array}{rrr}\vdots & \cdots & \vdots \\ & \ddots & \vdots \\ & \cdots & \end{array}\right]\left\{\begin{array}{l}\text { if } i, j \text { is relashion } A_{i, j}=1 \\ O W, \quad A_{i, j}=0\end{array}\right.$

After calculation A, by solving (8) Matrix $W$ will be obtained and then, by using (7) the new features will be obtained in new space (Liao et al, 2016a). Finally, the fused features $\mathrm{Z}$ in the lowerdimensional subspace will be utilized as an input to do classification.

\subsection{Expression of the proposed method}

In this section, a feature reduction method will be proposed with the LBG which Fig1 expresses that. The first step in this algorithm is spectral-spatial features extraction from 3D-Gabor filters. In this step, spectral-spatial features will be extracted by the 3D-Gabor filters on the original hyperspectral data sets. Subsequently, features dimensions will be reduced by Local Binary Graph Analysis (LBG).

Suppose that $x_{i}^{3 D-G a b o r}$ denotes the spectral-spatial features extracted from 3D-Gabor filters after normalization of their values between interval $[0,1]$ where $x_{i}^{3 D-G a b o r} \in R^{D}$ that $\mathrm{D}$ is the number of dimensions. The goal of this method is to find a transformation matrix $W \in R^{D \times L}$ which reduced dimensionality (to d-dimensions) and fused features in a way of (10), simultaneously.

$$
z_{i}=W^{T} \cdot x_{i}^{3 D-G a b o r}
$$

Where $x_{i}^{3 D-G a b o r}$ is a multivariate parameter which can be set by $3 \mathrm{D}$-Gabors filters and $z_{i}$ is the feature fusion in lowerdimensional feature space with $z_{i} \in R^{L}$ and $L \leq \mathrm{D}$. Also, a usable way to compute the transformation matrix $\mathrm{W}$ can be 
defined by (8). Taking advantage of the fact that pixels within a spatial neighborhood are likely to share similar properties, matrix A will be constructed. The matrix $A \in R^{N \times N}$ represents the adjacency relation of all data points built on the features of the 3D-Gabor filter. Due to limitations in computing the $\mathrm{X}$ matrix, such as the high computational volume of this matrix, the following relationships will be used (Liao et al, 2016a):

$$
W^{T}\left(x^{3 D-G a b o r}\right) D^{F u s}\left(x^{3 D-G a b o r}\right) W=I
$$

Where $D^{F u s}$ is a diagonal matrix with $D_{i i}^{f u s}=\sum_{j=1}^{n} A_{i j}^{f u s}$ and I is the identity matrix. The transformation matrix $\mathrm{W}=$ $\left(w_{1}, w_{2}, \ldots, w_{r}\right)$ which is constructed of $\mathrm{r}$ eigenvectors with least eigenvalues $\lambda_{1} \leq \lambda_{2} \leq \cdots \leq \lambda_{r}$ (that obtained from generalized eigenvalue problem), will be computed:

$$
\begin{aligned}
& x^{3 D-G a b o r} \cdot L^{F u s} \cdot\left(x^{3 D-G a b o r}\right)^{T} \cdot w \\
& =\lambda \cdot x^{3 D-G a b o r} \cdot L^{F u s} \cdot\left(x^{3 D-G a b o r}\right)^{T} \cdot w
\end{aligned}
$$

Where $L^{F u s}=D^{F u s}-A^{F u s}$ is the fusion Laplacian Matrix. By solving this problem, transformation matrix $W \in R^{D \times L}$ will be obtained. In the LBG method (Liao et al, 2016a) the size of the sliding window has a significant influence on the preservation of local spatial neighborhood information (e.g., texture). In this paper, the sliding window had been set with a fixed average size and with changing $\mathrm{k}$ nearest neighbors, a satisfying result will be obtained.

\subsection{Feature Reduction}

After calculating the transformation matrix $\mathrm{W}\left(W \in R^{D \times L}\right)$ from (12), the features can be reduced by the LBG method from (10). In this step, $\mathrm{K}$ nearest neighbors will be found by simulation for each pixel within a $7 \times 7$ sliding window in the LBG method. Finally, the fused features from the high dimensional 3D-Gabor filters $x_{i}^{3 D-G a b o r} \in R^{D}$ will be obtained into a lowerdimensional subspace $z_{i} \in R^{L}$ with equation (10). Therefore, if the $3 \mathrm{D}-$ Gabors features have "D" dimension $\left(x_{i}^{3 D-\text { Gabor }} \in R^{D}\right)$ by applying to transform matrix "W" $\left(W \in R^{D \times L}\right)$, the features will be reduced to the "L" dimension through (10) that $L \leq \mathrm{D}$. Thus, by using graph theory (Bioucas-Dias et al, 2012a, Huang, 2013a, Liao et al, 2016a), the extracted features of the 3D-Gabor filter will be reduced. After spatial-spectral feature extraction and reduction by the proposed method, the fused features vector $\mathrm{Z}$ will be obtained in the lower-dimensional subspace as an input to do classification. By building a local binary graph within a sliding window, memory cost and computational complexity will be reduced much better than conventional methods, such as the global binary graph, in addition, local spatial neighborhood information will be preserved. The algorithm of the proposed method uses the LBG to reduce the dimensionality of spectralspatial extracted features for accurate classification.

\section{EXPERIMENTAL RESULTS}

\subsection{Hyperspectral Image Data Sets}

Experiments were run on two data sets, i.e. the 'Indian Pines' and 'University of Pavia'. The first data set was obtained by airborne visible/infrared imaging spectrometer (AVIRIS) over Northwestern Indiana in June 1992, with 200 spectral bands in the wavelength range $0.4-2.5 \mu \mathrm{m}$ and low spatial resolution of $20 \mathrm{~m}$ by pixel.
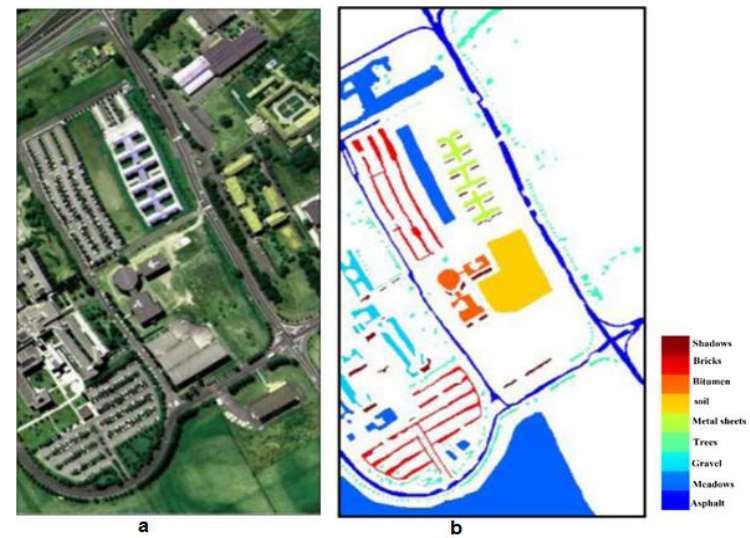

Figure 4. RGB map (a), Reference map (b) for Pavia University
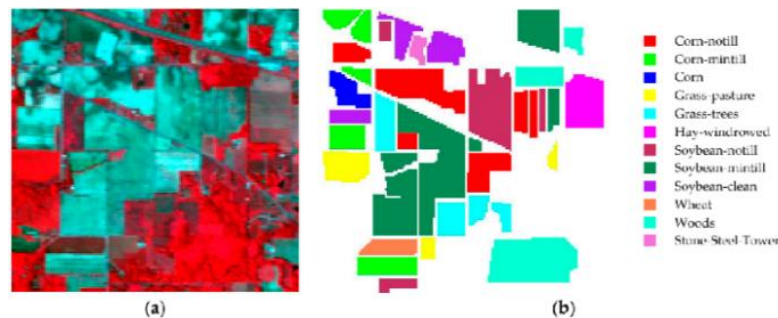

Figure 5. RGB map (a), Reference map (b) for Indian Pines

The calibrated data are available online (along with detailed ground-truth information) and the second data set was acquired over an urban area in the city of Pavia, Italy. The data were collected by the reflective optics system imaging spectrometer (ROSIS) sensor, with 115 spectral bands in the wavelength range $0.43-0.86 \mu \mathrm{m}$ and very fine spatial resolution of $1.3 \mathrm{~m}$ by pixel.

- Indian pines: All scene $(145 \times 145$ pixels $)$ includes 16 classes, ranging in size $20-2468$ pixels. All 200 bands (including some noisy bands) will be preserve to see the effect of noise on the classification. In our experiments, classes with less than 30 labeled pixels were removed, resulting in 14 classes with available labeled samples. Note that the color in the cell denotes different classes in the classification maps.

- University of Pavia: The image composed of $610 \times$ 340 pixels was collected over the University of Pavia, Italy, and contains 103 spectral channels after removal of noisy bands. This dataset includes nine land cover/use classes.

RGB map (a) and reference map (b) for Indian pines have been shown in fig 5. Also, RGB map (a) and reference map (b) for Pavia University dataset have been shown in fig 4 .

\subsection{Experimental Setup}

At first, to obtain spatial features from MP, the PCA (BioucasDias et al, 2012a, Huang, 2013a, Liao et al, 2016a) is applying to the original HS, and then the first 4 PCs for Indian pine and the first 3 PCs for the University area were selected to make the MP features. An incremental size circular SE of one to ten is applied to the image. Ten opening and ten closing operators were measured for each PC, resulting in an MP of 84 dimensions for Indian pine and 63 dimensions for the University area. Finally, the size of the neighborhood window (W) will be selected 15 and the number of K-Nearest neighbors will be selected 15, also. Furthermore, 3D-Gabor filters parameters will be chosen from the following table I. 
Table I. Parameters for 3D-Gabor

\begin{tabular}{|c|c|c|c|c|}
\hline parameters & \multicolumn{3}{|l|}{ Values } \\
\hline$\Theta$ & 0 & 45 & 90 & 135 \\
\hline$\phi$ & 0 & 45 & 90 & 135 \\
\hline $\mathrm{F}$ & $\ldots$ & $\ldots$ & 0.125 & .0625 \\
\hline
\end{tabular}

Which the size of Gabor Filter window is: $21 \times 21 \times 21$.

In all experiments, SVM classifier by 50 training samples with Polynomial kernel in MATLAB SVM toolbox, LIBSVM for classification is used. The classification results are quantitatively calculated by measuring the overall accuracy (OA), the average accuracy (AA), the kappa coefficient ( $\kappa$ ), and McNemar test (Z) (Ghamisi, 2014a) The analyses were carried out on 32-b, 1.40 $\mathrm{GHz}$ Intel i5-5930K (1 core) CPU computer with 32 GB memory.

\subsection{Results on Indian Pines Data Set and PaviaU Data Set}

All results were measured for the remaining labeled samples in the ground truth Also, all tests were calculated and averaged over 10 times and fifty samples per class were randomly selected.

Table II. Results on Indian Pines Data Set. $N_{f}$ is number of feature

\begin{tabular}{||c|c:c:c:c||}
\hline \hline Methods & $N_{f}$ & OA & AA & Kappa \\
\hline Hyperspectral & 200 & 71.6 & 84.8 & 72.5 \\
MP & 124 & 71.1 & 86.6 & 76 \\
Hyperspectral+ MP & 224 & 86.2 & 91.9 & 86.85 \\
3D-Gabor & 6400 & 87.6 & 92.4 & 90.2 \\
LBG & $\mathbf{4 0}$ & 83.5 & 90.9 & 85 \\
Proposed & $\mathbf{4 0}$ & $\mathbf{9 2 . 6}$ & $\mathbf{9 5 . 2}$ & $\mathbf{9 4 . 8}$ \\
\hline
\end{tabular}

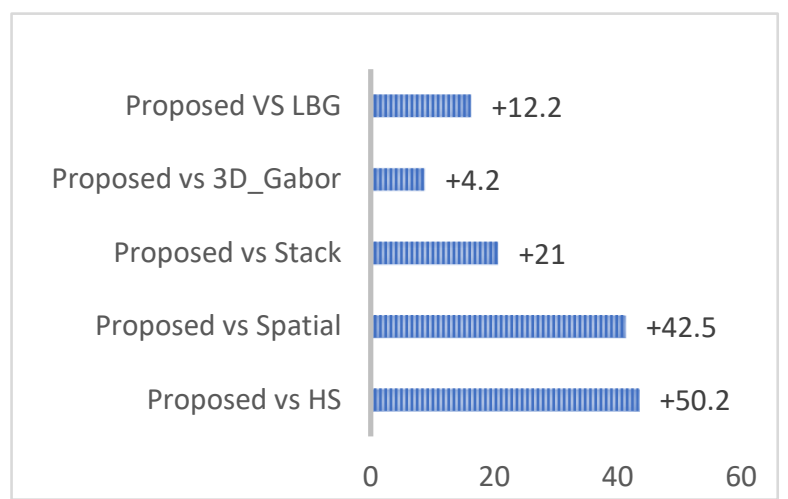

Figure 6. The McNemar test for proposed and other method

According to Table II: As can be seen, the best result is related to the proposed method Also, As we expected, the use of spatial and spectral information simultaneously improved the conclusion.

The closest conclusion to the proposed method is the 3D- Gabor method because the 3D-Gabor can extract spatial and spectral features simultaneously.

In the following, The McNemar test is shown in Fig. 6 to compare different methods and the proposed method (Fig. 6). At the end, the classification map for the several methods is presented in Fig.8.
Table III. Describes the classification's parameters of this data. Results on Pavia University data Set. $N_{f}$ is number of feature

\begin{tabular}{||c|c|c|c|c||}
\hline \hline Methods & $N_{f}$ & OA & AA & Kappa \\
Hyperspectral & 103 & 82.6 & 85.1 & 84.7 \\
MP & 104 & 78.5 & 84.1 & 71.9 \\
Hyperspectral+ MP & 207 & 88.5 & 89.3 & 87.9 \\
3D-Gabor & 3296 & 92.3 & 93.4 & 92.1 \\
LBG & $\mathbf{4 0}$ & 93.9 & 94.6 & 92.7 \\
Proposed & $\mathbf{4 0}$ & $\mathbf{9 6 . 2}$ & $\mathbf{9 6 . 7}$ & $\mathbf{9 4 . 6}$ \\
\hline
\end{tabular}

According to Table III: in this table, the proposed method has the best result. Furthermore, the use of spatial and spectral information simultaneously improved the results.

The closest conclusion to the proposed method is the LBG method.

In the following, the McNemar test is presented in Fig.7 for different methods and the proposed method in Fig.7. Also, the classification map for the several methods is presented in Fig.9.

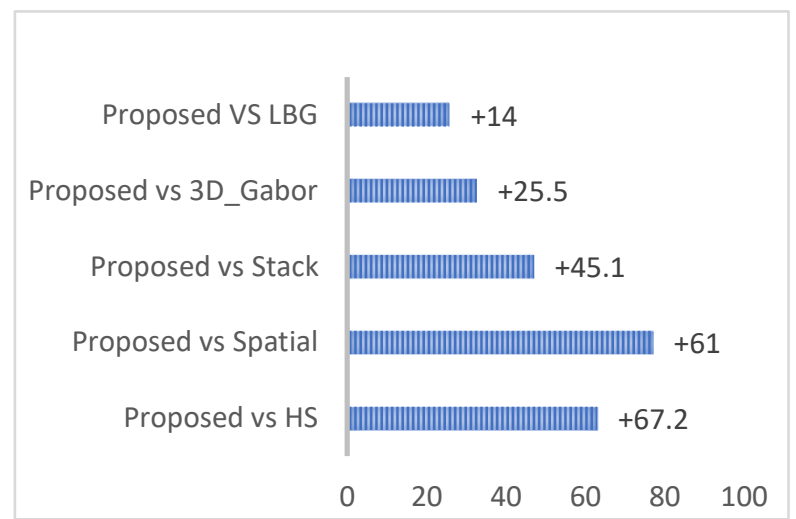

Figure 7. The McNemar test for proposed and other method

\section{CONClusion}

In this paper, a method to reduce features of spectral and spatial information was proposed. To that end, spectral and spatial features extracted from 3D-Gabor filters. At first, the spatial and spectral features extracted from 3D-Gabor filters. After then, a local binary graph method (LBG) was proposed to reduce the features. Therefore, the fusion features in lower-dimensional feature space will be obtained. By constructing a local binary graph within a sliding window, we not only reduce memory cost and computational complexity but also increase the preservation of local spatial neighborhood information. The algorithm of the proposed method uses LBG to dimension reduction of spectralspatial features for classification. Experiments were tested on two real dataset (Pavia University and Indian Pines). The experimental results on Indian Pines and Pavia University showed that the fusion of spatial and spectral features leads to improving the classification. Furthermore, Classification results on two real HS data show the efficiency of the proposed method. The best result for parameters of comparison in the classification related to the proposed method, so the overall accuracy is increased to 96.2, 92.6 for Pavia University and Indian Pines dataset. According to the results, the proposed method in this paper has the best results compared to other methods. 


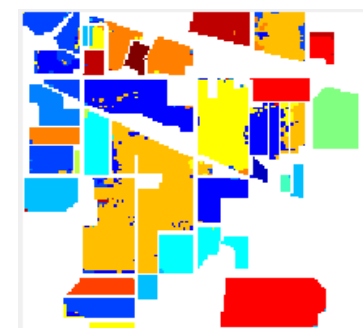

a
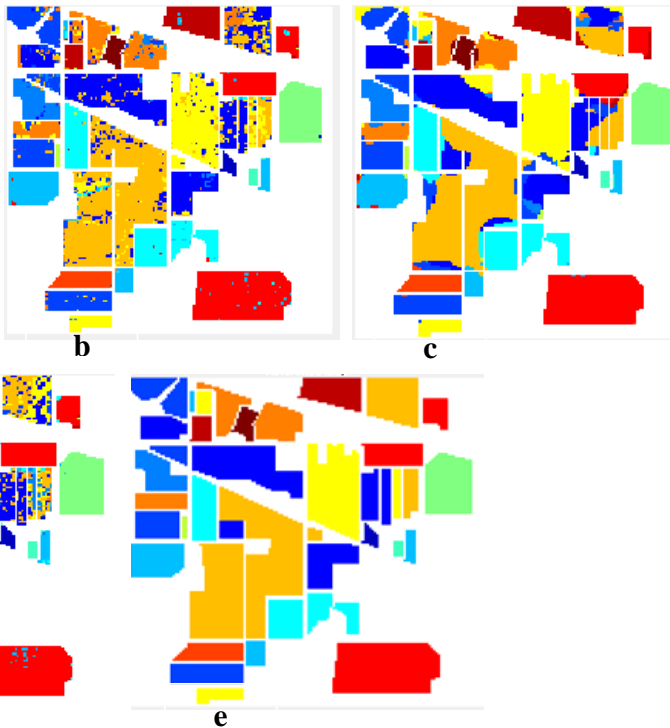

Figure 8. Classification maps for Indian Pines: (a) proposed, (b) LBG, (c) 3D-Gabor, (d) Stacking and (e) GTM
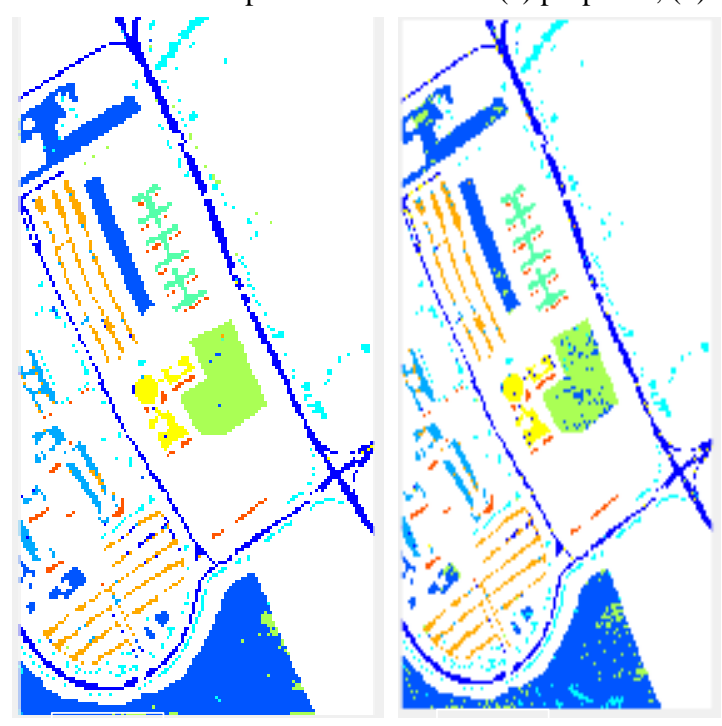

a
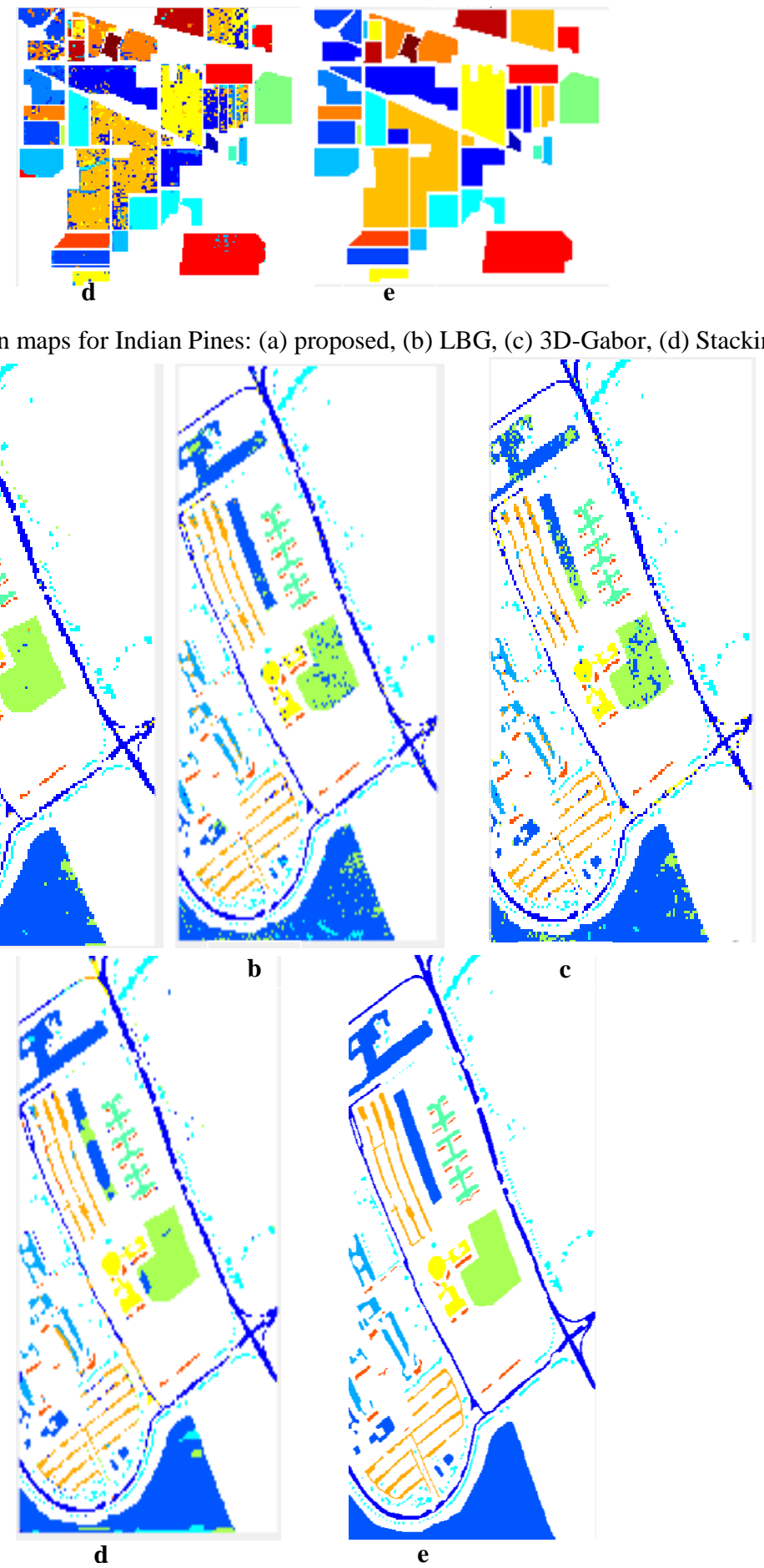

Figure9. Classification maps for Pavia U: (a) proposed, (b) LBG, (c) 3D-Gabor, (d) Stacking and (e) GTM 


\section{REFERENCES}

Benediktsson, J.A., Palmason, J.A. and Sveinsson, J.R., 2005. Classification of hyperspectral data from urban areas based on extended morphological profiles. IEEE Transactions on Geoscience and Remote Sensing, 43(3), pp.480-491.

Bioucas-Dias, J.M., Plaza, A., Dobigeon, N., Parente, M., Du, Q., Gader, P. and Chanussot, J., 2012. Hyperspectral unmixing overview: Geometrical, statistical, and sparse regression-based.

Chang, C.C. and Lin, C.J., 2011. LIBSVM: A library for support vector machines. ACM transactions on intelligent systems and technology (TIST), 2(3), p.27.

Chen, W., Zheng, R., Baade, P.D., Zhang, S., Zeng, H., Bray, F., Jemal, A., Yu, X.Q. and He, J., 2016. Cancer statistics in China, 2015. CA: a cancer journal for clinicians, 66(2), pp.115-132.

Fang, L., Li, S., Duan, W., Ren, J. and Benediktsson, J.A., 2015. Classification of hyperspectral images by exploiting spectralspatial information of superpixel via multiple kernels. IEEE transactions on geoscience and remote sensing, 53(12), pp.6663-6674

Fauvel, M., Tarabalka, Y., Benediktsson, J.A., Chanussot, J. and Tilton, J.C., 2012. Advances in spectral-spatial classification of hyperspectral images. Proceedings of the IEEE, 101(3), pp.652675.

Ghamisi, P., Benediktsson, J.A., Cavallaro, G. and Plaza, A., 2014. Automatic framework for spectral-spatial classification based on supervised feature extraction and morphological attribute profiles. IEEE Journal of Selected Topics in Applied Earth Observations and Remote Sensing, 7(6), pp.2147-2160.

Ghassemian H., Landgrebe, D., 1988a. On-line object feature extraction for multispectral scene representation, NASA Technical Report Server, TR-EE-88-34:

http://ntrs.nasa.gov/search.jsp?R=19900019572\&qs=Nm\%3D42935855 $63 \% 7$ CAuthor\%7CGhassemian\%2C\%2520Hassan\%26N\%3D0.

Ghassemian H., Landgrebe, D., 1988b. Object-Oriented Feature Extraction Method for Image Data Compaction, IEEE Control System Magazine, 8 (3), pp. 42-46.

Huang, X. and Zhang, L., 2012. An SVM ensemble approach combining spectral, structural, and semantic features for the classification of high-resolution remotely sensed imagery. IEEE transactions on geoscience and remote sensing, 51(1), pp.257272.

Hussain, S.A., Hasan, R. and Hussain, S.J., 2018. Classification and Detection of Plant Disease using Feature Extraction Methods. International Journal of Applied Engineering Research, 13(6), pp.4219-4226.

Imani, M. and Ghassemian, H., 2015. Feature reduction of hyperspectral images: discriminant analysis and the first principal component. Journal of AI and Data Mining, 3(1), pp.19.

Imani, M. and Ghassemian, H., 2015. Feature space discriminant analysis for hyperspectral data feature reduction. ISPRS Journal of Photogrammetry and Remote Sensing, 102, pp.1-13.

Imani, M. and Ghassemian, H., 2016, May. GLCM, Gabor, and morphology profiles fusion for hyperspectral image classification. In 2016 24th Iranian Conference on Electrical Engineering (ICEE) (pp. 460-465). IEEE.

Kianisarkaleh, A. and Ghassemian, H., 2016. Nonparametric feature extraction for classification of hyperspectral images with limited training samples. ISPRS Journal of Photogrammetry and Remote Sensing, 119, pp.64-78.

Kowkabi, F., Ghassemian, H. and Keshavarz, A., 2016, July. Hyperspectral endmember extraction and unmixing by a novel spatial-spectral preprocessing module. In 2016 IEEE International Geoscience and Remote Sensing Symposium (IGARSS) (pp. 3382-3385). IEEE.

Liao, W., Dalla Mura, M., Chanussot, J. and Pižurica, A., 2015. Fusion of spectral and spatial information for classification of hyperspectral remote-sensed imagery by local graph. IEEE Journal of Selected Topics in Applied Earth Observations and Remote Sensing, 9(2), pp.583-594.

Li, S. and Wei, D., 2013. Extremely high-dimensional feature selection via feature generating samplings. IEEE transactions on cybernetics, 44(6), pp.737-747.

Li, S. and Wei, D., 2013. Extremely high-dimensional feature selection via feature generating samplings. IEEE transactions on cybernetics, 44(6), pp.737-747.

Mirzapour, F. and Ghassemian, H., 2013. Using GLCM and Gabor filters for classification of PAN images, 21th Iranian conference, Mashhad, Iran; 14 - 16 May 2013.

Pal, M. and Foody, G.M., 2010. Feature selection for classification of hyperspectral data by SVM. IEEE Transactions on Geoscience and Remote Sensing, 48(5), pp.2297-2307

Rizanti, N.A. and Setyaningrum, A.H., 2016, April. Colon detection using Principal Component Analysis (PCA) and Support Vector Machine (SVM). In 2016 4th International Conference on Cyber and IT Service Management (pp. 1-7). IEEE.

Soille, P., 2013. Morphological image analysis: principles and applications. Springer Science \& Business Media.

Swaminathan and Madhukumar, R. and Madhukumar, A.S., 2017. Classification of error correcting codes and estimation of interleaver parameters in a noisy transmission environment. IEEE Transactions on Broadcasting, 63(3), pp.463-478.

Swaminathan, R. and Madhukumar, A.S., 2017. Classification of error correcting codes and estimation of interleaver parameters in a noisy transmission environment. IEEE Transactions on Broadcasting, 63(3),

Taghipour, A. and Ghassemian, H., 2017. Hyperspectral anomaly detection using attribute profiles. IEEE Geoscience and Remote Sensing, vol. 14, no. 7, pp. 1136-1140, 2017.

Zhang, Q., Tian, Y., Yang, Y. \& Pan, C. (2015). Automatic Spatial-Spectral Feature Selection for Hyperspectral Image via Discriminative Sparse Multimodal Learning. IEEE Transactions on Geoscience and Remote Sensing, vol. 53, no. 1, pp. 261-279. 